\title{
Temperature and Pressure Effects on the Separation Efficiency and Desorption Kinetics in the $\mathrm{NH}_{3}-\mathrm{CO}_{2}-\mathrm{H}_{2} \mathrm{O}$ System
}

\author{
Georgios Kolliopoulos and Vladimiros G. Papangelakis* \\ Department of Chemical Engineering and Applied Chemistry, University of Toronto \\ 200 College Street, Toronto, Ontario, Canada M5S 3E5 \\ (*Corresponding author: vladimiros.papangelakis@utoronto.ca)
}

\section{Supporting Information}

The comparison between experimental $\mathrm{CO}_{2}$ speciation and $\mathrm{pH}$ measurements $\left(\right.$ at $25^{\circ} \mathrm{C}$, 1.01 bar) with the OLI-MSE predicted values $\left(25^{\circ} \mathrm{C}, 1.01\right.$ bar $)$ in $\mathrm{NH}_{3}-\mathrm{CO}_{2}-\mathrm{H}_{2} \mathrm{O}$ system for all experimental runs (temperature, pressure) is presented in Figure S1. Table S1 presents the regressed overall desorption rate constants $\mathrm{k}\left(\mathrm{min}^{-1}\right)$ for $\mathrm{NH}_{3}$ and $\mathrm{CO}_{2}$ from the $\mathrm{NH}_{3}-\mathrm{CO}_{2}-\mathrm{H}_{2} \mathrm{O}$ DDS.

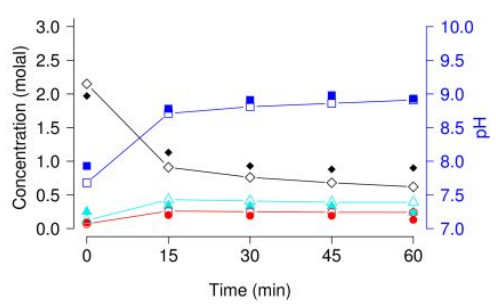

(a)

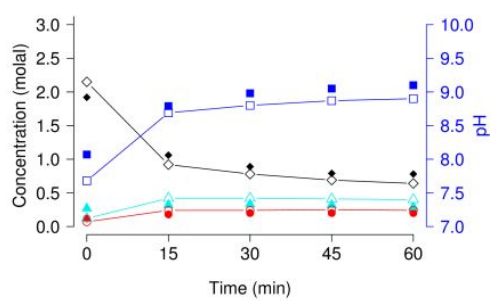

(b)

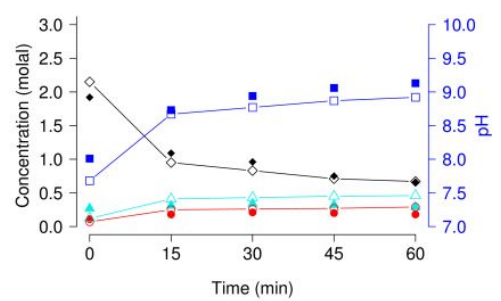

(c) 


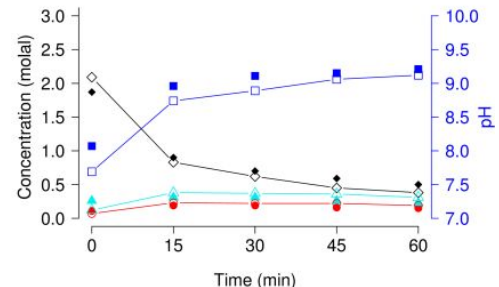

(d)

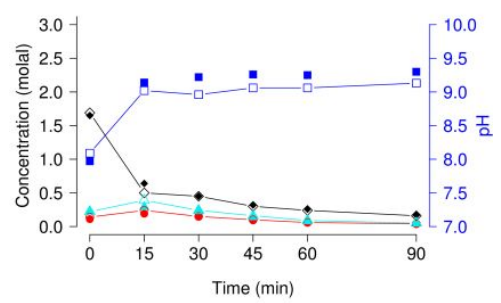

$(\mathrm{g})$

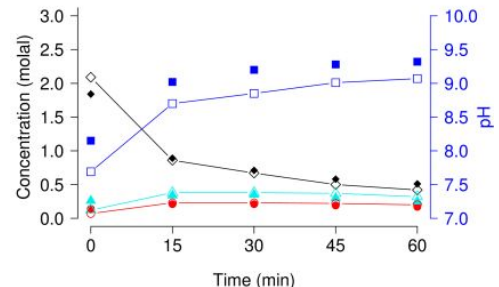

(e)

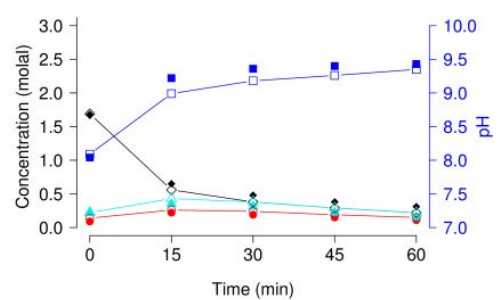

(h)

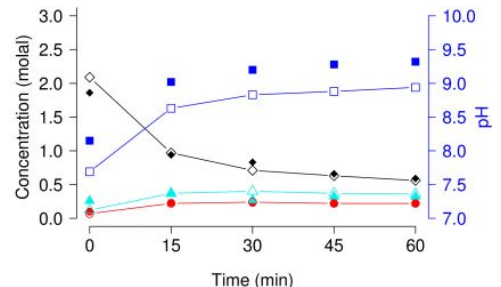

(f)

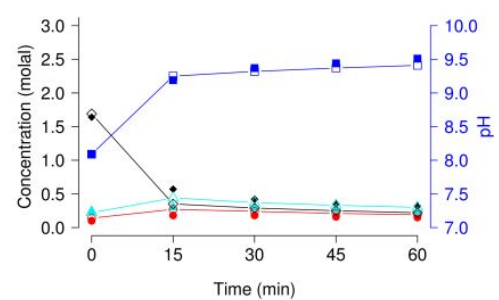

(i)

$\begin{array}{llll}\bullet \mathrm{HCO}_{3}^{-} & \bullet \mathrm{CO}_{3}^{2-} & \triangle \mathrm{NH}_{2} \mathrm{CO}_{2}^{-} & \square \mathrm{pH} \\ \diamond \mathrm{HCO}_{3}^{-}(\mathrm{OLI}) & \circ \mathrm{CO}_{3}^{2-}(\mathrm{OLI}) & \triangle \mathrm{NH}_{2} \mathrm{CO}_{2}^{-}(\mathrm{OLI}) & \square \mathrm{pH}(\mathrm{OLI})\end{array}$

Figure $\mathrm{S} 1$-Experimental $\mathrm{CO}_{2}$ speciation and $\mathrm{pH}$ in $\mathrm{NH}_{3}-\mathrm{CO}_{2}-\mathrm{H}_{2} \mathrm{O}$ (measured at $25^{\circ} \mathrm{C}, 1.01$ bar) vs. predicted values $\left(25^{\circ} \mathrm{C}, 1.01 \mathrm{bar}\right)$; experimental (temperature, pressure) in (a) $\left(30^{\circ} \mathrm{C}, 0.35\right.$ bar), (b) $\left(30{ }^{\circ} \mathrm{C}, 0.55\right.$ bar), (c) $\left(30{ }^{\circ} \mathrm{C}, 0.75\right.$ bar), (d) $\left(40{ }^{\circ} \mathrm{C}, 0.35\right.$ bar), (e) $\left(40{ }^{\circ} \mathrm{C}, 0.55\right.$ bar), (f) (40 ${ }^{\circ} \mathrm{C}, 0.75$ bar $),(\mathrm{g})\left(50^{\circ} \mathrm{C}, 0.35 \mathrm{bar}\right),(\mathrm{h})\left(50^{\circ} \mathrm{C}, 0.55 \mathrm{bar}\right)$, and (i) $\left(50{ }^{\circ} \mathrm{C}, 0.75\right.$ bar $)$ 
Table $\mathrm{S} 1$ - Overall desorption rate constants $\mathrm{k}\left(\mathrm{min}^{-1}\right)$ for the separation of $\mathrm{NH}_{3}$ and $\mathrm{CO}_{2}$ from an $\mathrm{NH}_{3}-\mathrm{CO}_{2}-\mathrm{H}_{2} \mathrm{O}$ DDS (15-60 min)

\begin{tabular}{|c|c|c|c|}
\hline \multicolumn{3}{|c|}{$\mathrm{NH}_{3}($ total dissolved $) \rightleftharpoons \mathrm{NH}_{3}(\mathrm{~g})$} & \multirow{2}{*}{$\frac{(\mathrm{R} 18)}{\mathrm{R}^{2}}$} \\
\hline $\mathrm{T}\left({ }^{\circ} \mathrm{C}\right)$ & $\mathrm{P}$ (bar) & $\mathrm{k}\left(\min ^{-1}\right)$ & \\
\hline 30 & 0.35 & $0.0031 \pm 0.0005$ & 0.9394 \\
\hline 40 & 0.35 & $0.0056 \pm 0.0004$ & 0.9540 \\
\hline 50 & 0.35 & $0.0182 \pm 0.0003$ & 0.9708 \\
\hline 30 & 0.55 & $0.0028 \pm 0.0003$ & 0.8545 \\
\hline 40 & 0.55 & $0.0048 \pm 0.0006$ & 0.9732 \\
\hline 50 & 0.55 & $0.0123 \pm 0.0010$ & 0.9051 \\
\hline 30 & 0.75 & $0.0003 \pm 3.6 \cdot 10^{-7}$ & 1.0000 \\
\hline 40 & 0.75 & $0.0042 \pm 0.0004$ & 0.8567 \\
\hline 50 & 0.75 & $0.0063 \pm 0.0005$ & 0.9001 \\
\hline \multicolumn{3}{|c|}{$\mathrm{CO}_{2}($ total dissolved $) \rightleftharpoons \mathrm{CO}_{2}(\mathrm{~g})$} & (R19) \\
\hline $\mathrm{T}\left({ }^{\circ} \mathrm{C}\right)$ & P (bar) & $\mathrm{k}\left(\min ^{-1}\right)$ & $\mathrm{R}^{2}$ \\
\hline 30 & 0.35 & $0.0054 \pm 0.0006$ & 0.9693 \\
\hline 40 & 0.35 & $0.0105 \pm 0.0003$ & 0.9987 \\
\hline 50 & 0.35 & $0.0198 \pm 0.0009$ & 0.9787 \\
\hline 30 & 0.55 & $0.0047 \pm 0.0007$ & 0.9613 \\
\hline 40 & 0.55 & $0.0100 \pm 0.0002$ & 0.9987 \\
\hline 50 & 0.55 & $0.0168 \pm 0.0005$ & 0.9980 \\
\hline 30 & 0.75 & $0.0041 \pm 0.0013$ & 0.8021 \\
\hline
\end{tabular}


0.9918 